\title{
Dyspnea After Tracheostomy for Painful Cervical Swelling: What is Your Diagnosis?
}

\author{
Rachidi Alaoui Siham ${ }^{1,2}$, Rkain ilham ${ }^{1,3 *}$, Touihmi Safaa ${ }^{3}$ and Motiaa Youssef $f^{1,4}$ \\ ${ }^{1}$ Faculty of Medicine and pharmacy of tangier, Abdelmalek Saadi University, Tétouan, Morocco \\ ${ }^{2}$ Department of radiology, university hospital, Tanger, Morocco \\ ${ }^{3}$ Department of otorhinolaryngology, Head and Neck surgery, university hospital, Tanger, Morocco \\ ${ }^{4}$ Department of anesthesiology and intensive care, university hospital, Tanger, Morocco
}

*Corresponding author: Rkain Ilham, Department of otorhinolaryngology, Head and Neck Surgery, university hospital, Tangier, Faculty of Medicine and pharmacy of tangier, Abdelmalek Saadi University, Tétouan, Morocco.

Received Date: August 05, 2020

Published Date: September 01, 2020

Case Report

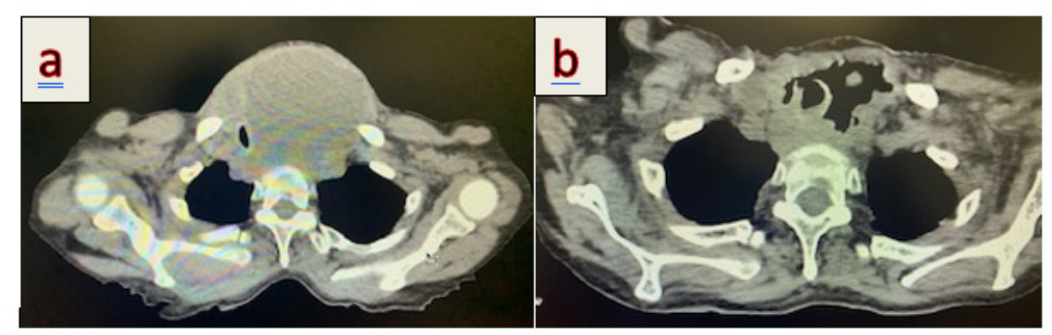

a: a left lobar thyroid mass with a liquid component compressing and driving back the trachea on the right with loss of separation border. b: Continuity solution of the trachea facing the site of the thyroid lesion

Figure 1: Cervico-thoracic CT in axial sections.

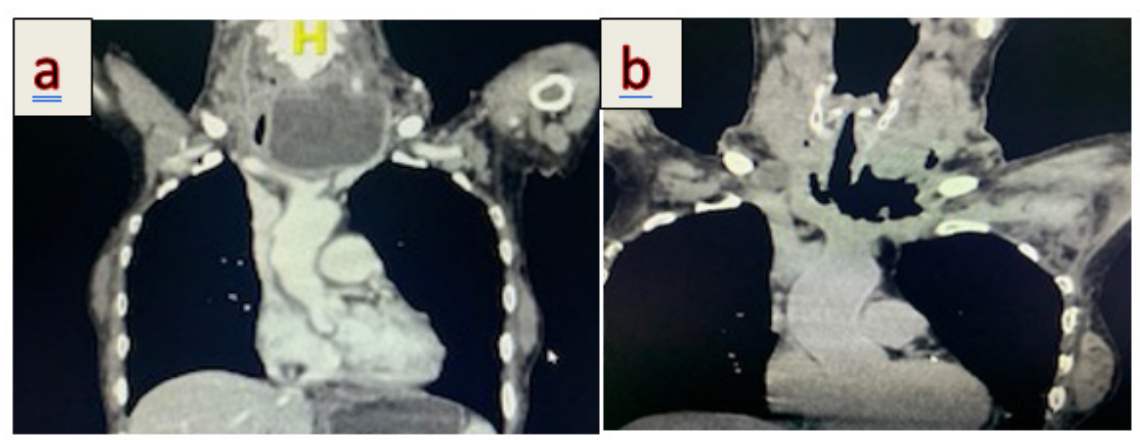

a: a left lobar thyroid mass with a liquid component compressing and driving back the trachea on the right with loss of separation border. b: Continuity solution of the trachea facing the site of the thyroid lesion.

Figure 2: Cervico-thoracic CT in coronal sections. 
A60-year-old women was irradiated for oesophageal carcinoma one year ago. The patient has a cervical mass for 3 months, gradually increasing in volume, associated with high dysphagia and intermittent dysphonia. One month later, the symptoms were aggravated by the installation of an inspiratory dyspnea that became both inspiratory and expiratory. Physical examination finds a $10 \mathrm{~cm}$ hard fixed and painful anterior cervical swelling ascending to swallowing, Nasofibroscopy finds a cordial diplegia

in closure without individualization of tumor process. At the end of these symptoms, the patient had a cervico-thoracic CT scan. An emergency tracheostomy with surgical biopsy was performed in another hospital structure. The patient was lost to follow-up for two months, then she presented to the emergency room for dyspnea, hence the realization of a second cervico-thoracic CT scan (Figure 1,2).

\section{Discussion}

Anterior tracheal rupture is rare or even exceptional. It can be post-traumatic or due to tumor invasion. Surgical treatment and primary reconstructive method for tracheal defect from invasion by differentiated thyroid carcinoma was reported by Atsumori, Liu H, Lifante, et al. [1-3]. In the case of our patient, biopsy with anatomopathological examination revealed an indifferenciated thyroid carcinoma type anaplastic, hence the aggressiveness of the invasion of the anterior wall of the trachea (due to a weakening and lysis of the wall). Some authors have also reported the effect of radiotherapy on the occurrence of tracheal fistula during cancers of the cervical esophagus, by necrosis of the posterior wall of the trachea [4].

\section{Acknowledgement}

None.

\section{Conflict of Interest}

No conflict of interest.

\section{References}

1. Atsumori Hamahata, Takeshi Beppu, Takashi Yamaki, Hiroyuki Sakurai (2018) Primary reconstructive method for tracheal defect from invasion by differentiated thyroid carcinoma. Auris Nasus Larynx 45(2): 371-376.

2. Liu H, Li Z, Dong H, Leng H, Sun H, et al. (2014) Surgical treatment and reconstruction of thyroid carcinoma invading cervical esophagus and trachea full-thickness 28(24):1927-1930.

3. Lifante JC, Mancini F, Mithieux F, Berger N, Latreche A, et al. (2001) Results of tracheal or esophageal resections in invasive thyroid carcinomas. Ann Chir 126(3): 236-241.

4. Jose M Soliz, Dam Thuy Truong, January Y Tsai, Angela T Truong (2018) Images in Anesthesiology: Tracheopharyngeal Fistula from Treated Hypopharyngeal Carcinoma. Anesthesiology 128(2): 386. 